\title{
Psychiatrie im Spannungsfeld zwischen Gefahrenabwehr und Therapie: Zwangsbehandlung in der Zeit nach der Rechtsprechung des Bundesverfassungsgerichts und des Bundesgerichtshofs
}

\author{
Psychiatry Caught Between Emergency Response and Therapy: Compulsory Treatment Following \\ the Jurisprudence of the Federal Constitutional Court and the Federal Supreme Court
}

Autoren

Institute
Jürgen L. Müller ${ }^{1}$, Peter Falkai², Frank Schneider ${ }^{3}$, Iris Hauth ${ }^{4}$, Wolfgang Maier ${ }^{5}$

Die Institute sind am Ende des Artikels gelistet.

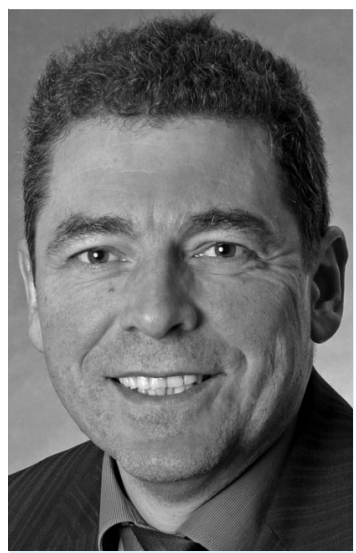

Prof. Jürgen L. Müller

\section{Bibliografie}

Dol http://dx.doi.org/ 10.1055/s-0033-1349560 Psychiat Prax 2013; 40: 365-367

(c) Georg Thieme Verlag KG Stuttgart · New York ISSN 0303-4259

Korrespondenzadresse Prof. Dr. med. Jürgen L. Müller Abteilung für Forensische Psychiatrie und Psychotherapie, Klinik für Psychiatrie und Psychotherapie

Rosdorfer Weg 70

37081 Göttingen

ju.mueller@asklepios.com
Mit Beschluss des Bundesverfassungsgerichts (BVerfG) vom 23.03.2011 (2 BVR 882 -09) wurde das Maßregelvollzugsgesetz Rheinland-Pfalz für verfassungswidrig erklärt, soweit es Behandlungen und Untersuchungen zur Erreichung des Vollzugsziels ohne Einwilligung des untergebrachten Patienten zuließ. Dem Gesetzgeber wurde auferlegt, verfassungskonforme rechtliche Grundlagen für eine medikamentöse Zwangsbehandlung $\mathrm{zu}$ schaffen. Angesichts der Schwere des Eingriffs in die Grundrechte des Betroffenen knüpfte das BVerfG die Zulässigkeit einer medikamentösen Zwangsbehandlung an hohe rechtliche Hürden. In der Konsequenz verwarf der Bundesgerichtshof 2012 (XII ZB 99/12; XII ZB 130/12) auch die betreuungsrechtliche Grundlage für eine Zwangsbehandlung. In diesen Entscheidungen waren Übergangsregelungen nicht vorgesehen, es entstand dringender Handlungsbedarf. Die DGPPN begrüßte in ihrer Stellungnahme vom 16.01.2012 [1], dass das BVerfG mit diesem Beschluss die Patientenautonomie gestärkt hat. Zugleich stieß die DGPPN eine grundsätzliche Diskussion über den Umgang mit psychisch Kranken an, die von ordentlichen Gerichten zur Sicherung psychiatrischer Einrichtungen überantwortet werden, aber krankheitsbedingt einer wirksamen Behandlung nicht zustimmen können. Die Konsequenzen einer solchen Nichtbehandlung eigen- oder fremdgefährdender Patienten für alle Beteiligten und die Gesellschaft wurden aufgezeigt. Die DGPPN begleitete die nicht zuletzt hierdurch angestoßene Auseinandersetzung in weiteren Stellungnahmen und wirkte auf die verantwortliche und praktikable Umsetzung der Neuregelung des Betreuungsrechts hin. Dieses trat am 26.02.2013 in Kraft [2] und wurde seitens der DGPPN uneingeschränkt wegen der damit verbundenen größeren Patientenautonomie und Rechtssicherheit für die Behandelnden begrüßt [3].

Die DGPPN wurde kritisiert [4 - 7], einerseits, weil sie die Zuständigkeit der Psychiatrie für Siche- rungsaufgaben ohne Behandlungsauftrag infrage stellte, andererseits, weil sie Zwangsmaßnahmen und Zwangsbehandlungen nicht sofort als einer zeitgemäßen psychiatrischen Patientenversorgung unangemessen verwerfen wollte. Unterstellt wurde, die DGPPN wolle den früheren Status quo nicht ändern, sondern rechtfertigen [7]. Gerade so, als stellte sich die DGPPN der Fortentwicklung einer Psychiatrie entgegen, welche die freie Willensbestimmung ihrer Patienten anerkennt, angemessen respektiert und für ihre Autonomie streitet. Wie sehr diese Polemik die Diskussion innerhalb der Fachgesellschaft um eine differenzierte Position verfehlt, verdeutlichen die verschiedenen Positionspapiere und Task Forces, mit denen die DGPPN ihre Herausforderung für die Fortentwicklung des Faches annimmt $[8,9]$. In verschiedenen Aufsätzen hat Zinkler für den Erfolg seines zwangs(behandlungs)freien Umgangs mit psychisch kranken Patienten geworben und zur Nachahmung aufgefordert [4-6]. Ebenso wies Finzen auf den nachholenden Charakter der aktuellen Diskussion in Deutschland hin und mahnte zur Gelassenheit [7]. Dies mag man so sehen, für die angestoßene Auseinandersetzung um die Aufgabe der Psychiatrie und den Umgang einer freiheitlichen Gesellschaft mit psychisch Kranken greifen diese Positionen allerdings zu kurz. Wie sehr, wird daran deutlich, dass Psychiatrie nicht nur kurative Aufgaben hat, also nicht nur behandlungsmotivierten Patienten Hilfe bietet, sondern, und das betrifft den Kern schwerer psychischer Störungen, auch und gerade dann gefordert ist, wenn die Willensbildung störungsbedingt vorübergehend beeinträchtigt ist.

Was also in Situationen, in denen anvertraute psychisch kranke Patienten, für die wir Sorge tragen, eigen- oder fremdgefährdend sind? Wenn Ärzte Aufgaben für die Gesellschaft wahrnehmen und von Gerichten beauftragt werden, durch Therapien Gefährdungen abzuwenden? Wie differenziert dies juristischerseits gesehen wird, be- 
leuchten aktuelle Gesetze und Gesetzesentwürfe in Hamburg, Baden-Württemberg und Niedersachsen, die eine Zwangsbehandlung bei Fremd- und Selbstgefährdung an unterschiedlich hohe Hürden binden.

Der angenommene selbstbestimmte freie Wille entscheidet über die Art und Dauer einer jeden Behandlung. Auch wenn eine psychische Störung die Willensbildung aufhebt, wenn Denkvorgänge verändert sind oder wahnhafte Ideen das Erleben bestimmen, ist eine Zwangsbehandlung nur in Ausnahmefällen zu diskutieren, nämlich dann, wenn aufgrund dieser psychischen Störung die Gesundheit oder gar das Leben des Betroffenen selbst bzw. das Leben und/oder die Gesundheit anderer erheblich gefährdet sind. Einen aufgrund eines Vergiftungswahns weder Nahrung noch Flüssigkeit zu sich nehmenden Patienten mit einer wahnhaften Depression in seinem lebensbedrohlichen Zustand zu belassen, ist mit unserem ärztlichen Selbstverständnis nicht zu vereinbaren. Hier geht es nicht um einen freiwillig fastenden Asketen, sondern um einen Patienten, der aufgrund einer schweren Erkrankung jegliche Nahrungsaufnahme verweigert. Ohne juristische Grundlage ist Hilfe nicht möglich.

Die DGPPN begrüßt die Gesetzesregelung zur betreuungsrechtlichen Einwilligung in eine ärztliche Zwangsmaßnahme, da sie den behandelnden Psychiater nicht mit der Entscheidung über eine Zwangsbehandlung alleine lässt, sondern diese in ein rechtsstaatliches Verfahren einbettet [2, 3]. Damit wird die Autonomie des Patienten gewahrt und sein Anspruch auf eine Behandlung geschützt [8].

Noch problematischer wird es, wenn aufgrund einer psychischen Störung fremdgefährdende Patienten psychiatrischen Kliniken überantwortet werden. Damit wird der Psychiatrie zusätzlich zu ihrer kurativen auch eine ordnungspolitische Aufgabe für die Gesellschaft übertragen. Diese gesellschaftliche Aufgabe bringt die Psychiatrie in ein Dilemma: Der gegen seinen Willen und aus Schutzgründen zugewiesene Patient ist oft nicht behandlungsmotiviert, sondern eine Gefahr für Mitpatienten, Personal und sich selbst.

Die Diskussion um die rechtliche Möglichkeit einer Zwangsbehandlung ist in einer zeitgemäßen Psychiatrie essenziell, sie muss aber zugleich dazu führen, dass die Möglichkeit einer Zwangsbehandlung zwar besteht, diese aber so selten wie möglich genutzt wird. Jede Zwangsmaßnahme ist eine erhebliche Belastung des Betroffenen und seiner Angehörigen und ein schwerwiegender Eingriff in dessen Persönlichkeitsrechte. Zwangsmaßnahmen sind selten notwendig und bedürfen selbstverständlich der richterlichen und gesellschaftlichen Kontrolle. Vermeidbare Zwangsmaßnahmen sind niemals zu rechtfertigen. Die DGPPN plädiert dafür, alle baulichen, therapeutischen und personellen Mittel auszuschöpfen, die die Anwendung von Zwangsmaßnahmen entbehrlich machen. Hierzu gehören die Respektierung von Patientenverfügungen und Behandlungsvollmachten, deeskalierende Gesprächstechniken, bauliche Gestaltung der Kliniken mit hinreichender Bewegungsmöglichkeit, individualisierte Behandlungsstrategien, Einbeziehung von Vertrauenspersonen, häufig auch Einsatz von viel Personal.

Die DGPPN kämpft gegen vermeidbaren Zwang bei der Behandlung und Betreuung psychisch Kranker und fördert die Ausbildung mit deeskalierenden Strategien, fordert aggressionslindernde Behandlungsatmosphären und Stationsgestaltungen und eine aufgabengerechte Personalausstattung. Die DGPPN unterstützt die Entwicklung gewaltfreier psychiatrischer Behandlungs- und Versorgungsstrukturen.
Weitere gravierende Probleme sind dagegen bislang kaum erwähnt: Das BVerfG bindet die Zwangsmedikation an das Ziel der Wiederherstellung der Einsichtsfähigkeit. Dies schließt eine relevante Anzahl von Patienten, die von einer Medikation profitieren können, aus. Nach der gegenwärtigen Rechtslage können Zwangsbehandlungen nur nach einer Unterbringung durchgeführt werden. Dies gilt auch für die Behandlung somatischer Krankheiten bei Einwilligungsunfähigen. Was also tun bei einem Demenzkranken mit einer behandlungsbedürftigen Erkrankung? Weder muss zur Behandlung auf jeden Fall untergebracht werden, noch ist zu erwarten, dass die mit der Demenz verbundene Einwilligungsunfähigkeit nach der Behandlung wiederhergestellt wird. Die DGPPN fordert den Gesetzgeber auf, gerade auch für die Versorgung somatischer erkrankter, dauerhaft einwilligungsunfähiger Patienten eine angemessene rechtliche Grundlage $\mathrm{zu}$ schaffen.

\section{Fazit}

$\nabla$

Die DGPPN bekennt sich zur Gesamtverantwortung für alle Patienten und warnt vor einer Entwicklung, die Patienten wirksame Hilfen vorenthält.

Die DGPPN bekennt sich auch zur geeigneten Behandlung und Betreuung der Patienten, die aufgrund ihrer Störung meistens vorübergehend, seltener andauernd zur Gefahr für sich selbst und für andere wie Angehörige, Mitpatienten, Betreuer und die Öffentlichkeit geworden sind. Verfügbare Behandlungsangebote, die gerade auch Schwerstkranken die gesellschaftliche Teilhabe wieder ermöglichen, dürfen nicht vorenthalten werden.

Die DGPPN begrüßt die Zielsetzung der UN-Behindertenkonvention, welche die Gleichstellung psychisch Behinderter wie deren Autonomie und Teilhabemöglichkeit fordert. Kein psychiatrischer Patient darf aufgrund seiner Erkrankung benachteiligt oder ausgeschlossen werden.

Institute

Abteilung für Forensische Psychiatrie und Psychotherapie, Klinik für Psychiatrie und Psychotherapie der Universitätsmedizin Göttingen

${ }^{2}$ Klinik für Psychiatrie und Psychotherapie am Klinikum der Universität München

${ }^{3}$ Medizinische Fakultät der RWTH Aachen, Klinik für Psychiatrie und Psychotherapie am Universitätsklinikum Aachen

${ }^{4}$ Klinik St. Joseph-Krankenhaus, Berlin-Weißensee

${ }^{5}$ Klinik und Poliklinik für Psychiatrie und Psychotherapie am Universitätsklinikum Bonn

\section{Literatur}

1 Müller J, Saimeh N, Nedopil N, Schneider F, Falkai P. Zum Urteil des Bundesverfassungsgerichts vom 23.03.2011 zur Zwangsbehandlung im Maßregelvollzug. Stellungnahme der Deutschen Gesellschaft für Psychiatrie, Psychotherapie und Nervenheilkunde (DGPPN). Stellungnahme Nr. 01 (16.01.2012). Im Internet: http://www.dgppn.de/publikatio nen/stellungnahmen/detailansicht/browse/1/select/stellungnahmen2012/article/141/zum-urteil-d-1.html (Stand: 26.08.2013)

2 Gesetz zur Regelung der betreuungsrechtlichen Einwilligung in ärztliche Zwangsmaßnahmen. Bundesgesetzblatt Jahrgang 2013 Teil I Nr. 9 (25.02.2013). Im Internet: http://www.bmj.de/SharedDocs/Down loads/DE/pdfs/Verkuendung_BGBl_Zwangsbehandlung.pdf?_blob= publicationFile (Stand: 26.08.2013)

3 Gesetz zur Regelung der betreuungsrechtlichen Einwilligung in eine ärztliche Zwangsmaßnahme bringt Klarheit. Stellungnahme der DGPPN Nr. 2 (31.01.2013). Im Internet: http://www.dgppn.de/publi kationen/stellungnahmen/detailansicht/browse/1/article/141/gesetzzur-r.html (Stand: 26.08.2013)

4 Zinkler M. Aufforderung zum Rechtsbruch? Die Stellungnahme der Deutschen Gesellschaft für Psychiatrie, Psychotherapie und Nerven- 
heilkunde zum Urteil des Bundesverfassungsgerichts zur Zwangsbehandlung im Maßregelvollzug vom 23.03.2011.. Recht und Psychiatrie 2012; 30: $62-63$

5 Zinkler M. Neuregelung von Zwang - ein Auftrag für die Fachgesellschaft? Psychiat Prax 2013; 40: 115-116

6 Zinkler M. Zwangsmedikation: Nach den Entscheidungen des Bundesverfassungsgerichts und des Bundesgerichtshofs zur Zwangsbehandlung - drei Fallberichte. Recht und Psychiatrie 2013; 2: 76-79

7 Finzen A. Zwangsmedikation: Die Psychiatrie nach den Urteilen - und davor. Recht und Psychiatrie 2013; 2: 71-75

8 Steinert T, Müller JL, Finzen A, Gründer G, Pollmächer T, Simon A, Spengler A, Maier W, Falkai P. Memorandum der DGPPN zur Autonomie und
Selbstbestimmung von Menschen. Stellungnahme Nr. 15 (18.10.2012) Im Internet: http://www.dgppn.de/publikationen/stellungnahmen/ detailansicht/select/stellungnahmen-2012/article/141/memorandum -d.html (Stand: 26.08.2013)

9 Herpertz SC, Maier W, Müller J, Nedopil N, Schneider F, Falkai P. Zum Beschluss des Bundesgerichtshofs vom 20.06.2012. Veröffentlicht am 17.07.2012. Stellungnahme der Deutschen Gesellschaft für Psychiatrie, Psychotherapie und Nervenheilkunde (DGPPN). Stellungnahme Nr. 13 (10.08.2012). Im Internet: http://www.dgppn.de/publikationen/stel lungnahmen/detailansicht/select/stellungnahmen-2012/article/141/ zum-beschlus-2.html (Stand: 26.08.2013) 